\title{
Lifetime Trauma and Depressive Symptomatology Among Older American Indians: The Native Elder Care Study
}

\author{
Authors: Ebru Cayir, Michael P. Burke, Mindi \\ Spencer, Mark B. Schure, and R. Turner Goins
}

The final publication is available at Springer via https://dx.doi.org/10.1007/s10597-017-0179-7

Cayir, Ebru, Michael P. Burke, Mindi Spencer, Mark B. Schure, and R. Turner Goins. "Lifetime Trauma and Depressive Symptomatology Among Older American Indians: The Native Elder Care Study." Community Mental Health Journal (November 2017): 1-8. DOI: 10.1007/ s10597-017-0179-7. 


\title{
Lifetime Trauma and Depressive Symptomatology Among Older American Indians: The Native Elder Care Study
}

\author{
Ebru Çayır ${ }^{1} \cdot$ Michael P. Burke $^{2} \cdot$ Mindi Spencer $^{1} \cdot$ Mark B. Schure $^{3} \cdot$ R. Turner Goins ${ }^{4}$ \\ 1 Department of Health Promotion, Education and Behavior, \\ Arnold School of Public Health, University of South \\ Carolina, 915 Greene St., Columbia, SC 29208, USA \\ 2 Food and Nutrition Service, U.S. Department of Agriculture, \\ 3101 Park Center Dr., Alexandria, VA 22302, USA \\ 3 Department of Health and Human Development, \\ Montana State University, P. O. Box 173540, Bozeman, \\ MT 59717-3540, USA \\ 4 Department of Social Work, College of Health and Human \\ Sciences, Western Carolina University, 4121 Little Savannah \\ Rd., Cullowhee, NC 28723, USA
}

\begin{abstract}
We examined the association between lifetime traumatic events with or without trauma response symptoms and depressive symptomatology in American Indians aged $\geq 55$ years from a tribe in the Southeastern US $(\mathrm{N}=362)$. Twenty-three percent of the sample experienced a traumatic event without trauma-response symptoms, whereas $14 \%$ experienced a traumatic event with at least one trauma-response symptom. After adjustment for sociodemographic characteristics and social support, participants who experienced a traumatic event with one or more trauma-response symptoms had higher odds of clinically relevant depressive symptomatology compared to (1) those who never experienced a traumatic event [odds ratio (OR) 3.2, $\mathrm{p}<0.05$ ], (2) and those who experienced a traumatic event without further symptoms (OR 3.7, p < 0.05). Our results suggest that mental health providers who serve older American Indians should consider the experience of traumatic events followed with response symptoms as a potential risk factor for further disruptions in psychological functioning.
\end{abstract}

Keywords American Indians · Older adults · Lifetime traumatic events · Depressive symptoms · Trauma response

\section{Introduction}

American Indians experience a higher lifetime incidence of traumatic events compared to other racial and ethnic groups in the US (Bassett et al. 2014; Beals et al. 2013; Ehlers et al. 2013; Manson et al. 2005). Approximately 22\% of American Indians in the US reside on tribal lands (US Census 2010) that are marked with extreme poverty, unemployment, and inadequate housing (Beals et al. 2013; Gone and Trimble 2012a). These socioeconomic risk factors are associated with interpersonal violence, childhood sexual abuse, homi-cide, suicide, and traffic accidents, which continue to affect the lives of many American Indians living on tribal lands (Brave Heart et al. 2012; Gone and Trimble 2012a; Manson et al. 2005; Robin et al. 1997). Furthermore, as recently as the 1950s, many American Indian children were forcibly removed from their families and placed in boarding schools that imposed western values while forbidding native lan-guages and spiritual practices, an experience that was trau-matic for many American Indians (EvansCampbell 2008; Whitbeck et al. 2004). Exposure to trauma among Ameri-can Indians is a public health concern, and understanding trauma's effect on health in this population has been called for by researchers and policy organizations (Brave Heart et al. 2012; Gone and Trimble 2012b; Goodkind et al. 2010). 
Exposure to traumatic events is associated with depressive disorders, anxiety, and post-traumatic stress disorder (PTSD) (Beals et al. 2013; Ehlers et al. 2013; Manson et al. 2005; Roh et al. 2014). A systematic review of the literature showed that among American Indians, PTSD is associated with experiences of traumatic events, and "the early, broad, and repeated risks or trauma and its sequelae" have been underscored (Bassett et al. 2014, p. 431). Of particular concern are older American Indians, as many are at an increased risk of experiencing a traumatic event in their lifetime (Cribbs et al. 2009; Roh et al. 2014; Whitbeck et al. 2004). Furthermore, if they did experience such an event, they may not have received adequate and culturally-tailored treatment due to barriers including unavailability of local services, lack of insurance, and mistrust towards mainstream mental health services (Gone and Trimble 2012b). Traumatic events experienced during childhood, and early adulthood are associated with mental disorders that last into the later years of life (Chapleski et al. 2004; Roh et al. 2015a). For example, older adults who witness an armed conflict, experience forced displacement and live in unfamiliar areas, or experience sexual, psychological and/or physical assault during earlier years of their life have increased risk for depression (Gameiro et al. 2014).

In the psychological trauma literature, the most commonly examined trauma response has been PTSD. However, there is a growing interest in understanding diverse representations of trauma response and their impact on mental health. Accordingly, partial or subthreshold PTSD diagnosis were developed to capture the experiences of individuals who do not report the complete range of PTSD symptoms following a traumatic event, but experience some of those symptoms and might benefit from treatment (Breslau et al. 2004; Marshall et al. 2001). Although definitions and criteria for subthreshold PTSD vary (Breslau et al. 2004; Marshall et al. 2001), the presence of partial trauma-response symptoms is associated with impaired psychological functioning (Marshall et al. 2001; Stein et al. 1997), indicating the importance of understanding nuances in trauma response, and how these nuances are related to mental health. Given the unique historical and contemporary experiences of trauma among American Indians, along with policy initiatives intended to reduce American Indian mental health disparities, it is important to investigate how variations in trauma response are related to mental health outcomes in this population (Gone and Trimble 2012b; Lillie-Blanton and Roubideaux 2005).

Previous work has examined the high incidence of traumatic events and PTSD in American Indians (Bassett et al. 2014; Beals et al. 2013). To our knowledge, however, no work has addressed the association between variations in trauma response and trauma-related mental health outcomes such as depression among older American Indians residing on tribal lands (Bassett et al. 2014; Chapleski et al. 2004; Roh et al. 2014, 2015b). Thus, the purpose of this study was to examine the association between lifetime traumatic events with or without trauma-response symptoms and depressive symptomatology among older members of a Southeastern American Indian tribe. Specifically, we used multivariable logistic regression to test the association between having clinically relevant depressive symptoms and experience of a lifetime traumatic event with or without trauma-response symptoms. We hypothesized that experiencing a traumatic event with trauma-response symptoms would be positively associated with clinically relevant depressive symptoms compared to never experiencing a traumatic event or experiencing a traumatic event without further trauma-response symptoms.

\section{Methods}

We used data from the Native Elder Care Study, a cross-sectional study of older American Indians (Goins et al. 2011). Interviewer-administered questionnaires were conducted between 2006 and 2008 with members of a federally recognized tribe in the Southeast region of the US. The inclusion criteria for the study were: being an enrolled tribal member with residence in the tribal service area, being non-institutionalized, being aged 55 years or older, and having passed a cognitive screening (Inouye et al. 1998). Based on tribal enrollment records, 1430 people were potentially eligible to participate. We used an age-stratified random sampling approach (55-64, 65-74 and 75 years or older) to select a total of 633 people to verify eligibility and request participation. Of these 633 people, 50 were deemed ineligible, and 78 refused to participate, yielding an $87 \%$ response rate and a final sample size of 505 . Men were more likely to decline participation than women (54 vs. $46 \%$, respectively, $\mathrm{p}<0.001)$. In addition to the approval obtained from West Virginia University Institutional Review Board (IRB), the Tribal IRB, the Tribal Council, and the Elder Council also approved the project.

\section{Measures}

\section{Dependent Variable}

Depressive symptomatology was measured using the Center for Epidemiologic Studies Depression Scale (CES-D) (Radloff 1977). The CES-D is a 20-item scale designed to assess depressive symptomatology in the previous week, and has been validated in the study population and other older American Indian populations (Chapleski et al. 1997; Schure and Goins, in press). CES-D scores can range from 0 to 60 , and a score $\geq 16$ is predictive of clinically relevant levels 
of depressive symptomatology (Radloff 1977). Therefore, we created a dichotomous CES-D variable (threshold 16) to assess the risk of clinical depression. The CES-D had a Cronbach's alpha of 0.87 in our sample.

\section{Independent Variable of Interest}

The independent variable of interest was experience of a traumatic event with or without indication of traumaresponse symptoms. We used a set of items from the National Anxiety Disorders Screening Day instrument to assess lifetime traumatic events and experience of PTSD symptoms (Ritsher et al. 2002). The main item that assessed lifetime traumatic events was "Have you ever had an extremely frightening, traumatic or horrible experience like being the victim of a violent crime, seriously injured in an accident, being sexually assaulted, seeing someone seriously injured or killed, or being the victim of a natural disaster?" Participants who affirmed the main item were then asked a sub-item to assess four trauma-response symptoms: (1) re-experiencing the traumatic event (e.g., through dreams, preoccupations or flashbacks); (2) emotional withdrawal or general loss of interest in important things; (3) troubles with sleeping, concentrating or temper; and (4) avoidance of places or things that remind of the traumatic event. An additional duration item asked whether any of these four symptoms lasted a month or longer. All items had response options of "no" or "yes."

Marshall and colleagues classified those who experienced a traumatic event and any of the trauma-response symptoms for at least a month as having subthreshold PTSD (Marshall et al. 2001). Following their approach, we assessed the prevalence of subthreshold PTSD, in addition to full PTSD. In our analytic sample, only 16 participants met the subthreshold PTSD criteria; therefore, we were not able to create a subthreshold or full PTSD variable that would be statistically reliable. We instead classified participants into three groups: (1) those who did not affirm the main trauma item; (2) those who affirmed the main trauma item only; and (3) those who affirmed the main trauma item and at least one of the four response symptoms or the duration item. This classification, although not capturing subthreshold or full PTSD, is still relevant because studies with diverse populations have documented positive associations between the experience of partial trauma-response symptoms and depressive symptomatology (Marshall et al. 2001; Roth et al. 2008; Stein et al. 1997).

\section{Potential Confounders}

We measured social support using the Medical Outcomes Study Social Support Survey (MOS-SSS) (Sherbourne and Stewart 1991). The MOS-SSS is a 19-item scale, with response options ranging from 1 (none of the time) to 5 (all of the time). Total scores range between 19 and 95, with higher scores indicating greater social support. The MOSSSS had a Cronbach's alpha of 0.95 in our sample. Socioeconomic and demographic variables included the participant's age (in years), gender (female vs. male), marital status (not married vs. married), total number of people living in the household (treated as continuous), and educational status ( $<12$ years vs. high school graduate/general educational diploma (GED), some college/associates degree, or college/ post-college graduate). Annual income was a categorical variable with a range of 1-10 corresponding to an annual income range of less than $\$ 4999-\$ 100,000$ or more. Annual income increased by $\$ 5000$ for each category $1-5 ; \$ 10,000$ for categories $6-7$; and $\$ 25,000$ for categories $8-10$.

\section{Statistical Analyses}

All estimates incorporated weights according to the agestratified sampling approach, and are representative of enrolled, non-institutionalized tribal members with residence in the tribal service area, aged 55 years or older, and without any cognitive impairment. We used multiple imputation via chained equations to account for missing data in the independent variable of interest and potential confounding variables. The income variable had the highest rate of missing data (27\%), followed by social support (19\%), and the remaining variables used in the analysis had less than $3 \%$ missing data. The imputation model incorporated all variables used in the analytic models (including the dependent variable); however, to reduce standard errors, the analytical models did not include participants who had missing values on the dependent variable (Von Hippel 2007). We used 50 imputed data sets, as the number of imputations conducted should be roughly equal to the percent of missing data across all variables (White et al. 2011). The analytic sample size with the imputed data was 362 . We found minimal differences between the estimates for weighted sociodemographic characteristics (i.e., age, household size, annual income, gender, marital status, and education) in the analytic sample and the original sample of 505 participants, suggesting that the analytic sample is representative of the original study population (analysis not shown).

Logistic regression models were used to test the association between the dichotomized CES-D variable and three categories of lifetime traumatic events. Model 1 was the unconditional model and included experience of trauma only. Model 2 adjusted for sociodemographic characteristics only, whereas Model 3 also included social support. We also tested whether there was a significant difference in CES-D scores between those who experienced a traumatic event without response symptoms and those who experienced at 
least one response symptom in the aftermath of the traumatic event. Estimates were considered significant at $\mathrm{p}<0.05$.

\section{Results}

The overall sociodemographic characteristics of the sample are as follows $(n=362)$ : the mean age was 65.9 (SE 0.27); the mean household size (persons) was 1.3 (SE 0.07); annual household income was \$15,000-\$24,999; a majority of participants were female (62.1\%), married (52.3 vs. $47.7 \%$ not married); $29.2 \%$ had less than 12 years of education, $31.9 \%$ had a high school diploma or GED, $30.5 \%$ had some college or associates degree, and $8.4 \%$ had a college or post-college graduate degree. The mean score on the MOS-SS was 81.5 (SE 0.82 ), and $15.7 \%$ of participants had a score greater than or equal to 16 of the CES-D. We also compared sociodemographic characteristics by experience of a traumatic event in the following order: no traumatic event experienced $(n=228)$; traumatic event without trauma-response symptoms $(n=84)$; and traumatic event with trauma-response symptoms $(n=50)$. Results are as follows, respectively: the mean ages were 67.1 (SE 0.43), 64.3 (0.77), and 62.8 (0.94); the mean household sizes (persons) were 1.3 (SE 0.08), 1.3 (0.14), and 1.5 (SE 0.18); annual household income were $\$ 10,000-\$ 14,999, \$ 15,000-\$ 24,999$, and \$10,000-\$14,999; a majority of participants were female $(65.6,55.2$, and $61.5 \%$ ), married (53.5 vs. $46.4 \%$ not married, 60.6 vs. $39.4 \%$ not married, and 66.3 vs. $33.7 \%$ not married); $31.1,20.8$, and $33.5 \%$ had less than 12 years of education, 35.0, 23.9, and $30.6 \%$ had a high school diploma or GED, 26.2, 43.3 and $29.8 \%$ had some college or associate degree, and 7.6, 12.0, and $6.0 \%$ had a college or post-college graduate degree; the mean scores on the MOS-SS were 82.8 (SE 0.89), 83.9 (SE 1.67), and 72.1 (3.05); and 11.7, 10.0, and $43.2 \%$ of participants had a score greater than or equal to 16 of the CES-D.

Across all logistic regression models, those who experienced a traumatic event with further symptoms had higher odds of clinically relevant depressive symptomatology compared to those who never experienced a traumatic event or those who experienced a traumatic event without further symptoms. Specifically, in Model 1, those with a traumatic experience with further symptoms had 5.7 times the odds [95\% confidence interval (CI) 2.65, 12.43, p < 0.001] of clinically relevant depressive symptomatology compared to those who never experienced trauma. Furthermore, a linear contrast showed that compared to the older adults with a traumatic experience without trauma-response symptoms, those with a traumatic experience with trauma-response symptoms had 6.9 times the odds $(95 \%$ CI for difference $2.36,20.01, \mathrm{p}<0.001)$ of clinically relevant depressive symptomatology. In Model 2, the odds were reduced such that those with further symptoms had 4.6 times the odds $(95 \%$ CI $2.02,10.57, \mathrm{p}<0.001)$ of clinically relevant depressive symptomatology compared to those who never experienced trauma. Furthermore, a linear contrast showed that compared to the older adults with a traumatic experience without trauma-response symptoms, those with a traumatic experience with trauma-response symptoms had 4.9 times the odds $(95 \% \mathrm{CI}$ for difference $1.71,14.36, \mathrm{p}<0.01)$ of clinically relevant depressive symptomatology. In Model 3 , the odds were further reduced: older adults with further symptoms had 3.2 times the odds (95\% CI 1.31, 7.93, $\mathrm{p}<0.05$ ) of clinically relevant depressive symptomatology compared to those who never experienced trauma. A linear contrast showed that compared to the older adults with a traumatic experience without trauma-response symptoms, those with a traumatic experience with trauma-response symptoms had 3.7 times the odds (95\% CI for difference $1.19,11.18, \mathrm{p}<0.05)$ of clinically relevant depressive symptomatology. Finally, among sociodemographic characteristics included in Models 2 and 3, only age and higher education were significantly associated with clinically relevant depressive symptomatology. Specifically, in Models 2 and 3, each 1-year increase in age was associted with 0.94 (95\% CI $0.90,0.98 ; \mathrm{p}<0.01)$ and $0.95(95 \%$ CI $0.90,0.99 ; \mathrm{p}<0.05)$ times the odss of clinically relevant depressive symptomatology. In Models 2 and 3, compared to those with less than 12 years of education, those with a college education or post-graduate education had $0.11(0.01,0.87 ; \mathrm{p}<0.05)$ and $0.10(95 \%$ CI $0.01,0.84: \mathrm{p}<0.05)$ times the odds of clinically relevant depressive symptomatology. In Model 3, each unit increase in MOS-SS score was associated with 0.96 (95\% CI $0.94,0.99 ; \mathrm{p}<0.01$ ) times the odds of clinically relevant depressive symptomatology.

\section{Discussion}

We examined the association between experience of traumatic events across the life course with or without traumaresponse symptoms and depressive symptomatology in a representative sample of older American Indians belonging to a tribe in the Southeastern US. Overall, we found significant differences in the odds of having clinically relevant depressive symptomatology as a function of experiencing a traumatic event followed with one or more response symptoms. Our findings are not only in line with previous research with American Indians regarding the adverse association between experiencing a traumatic event and depressive symptomatology (Beals et al. 2005, 2013; Chapleski et al. 2004; Robin et al. 1997) but also expand on this research in important ways.

Among participants who reported experiencing a traumatic event during their lifetime, only those who developed one or more trauma-response symptoms were more likely 
to experience clinically relevant depressive symptomatology. This finding supports the knowledge that although a majority of adults are exposed to at least one traumatic event during their lifetime, only a small proportion experiences traumarelated mental health conditions (Bonanno 2005). For example, people who were exposed to the same traumatic event at a specific time and place can have different trajectories of psychological functioning in the aftermath of the traumatic event, ranging from resilience (ability to maintain effective functioning in daily life and sense of well-being) to delayed recovery, or chronic disruptions in psychological functioning (Bonanno 2005; Eagle and Kaminer 2015).

One of the psychosocial resources that promote resilience in the aftermath of a traumatic event is social support. Similar to previous research, greater levels of social support appeared to be a protective factor among our sample. However, the association between the experience of traumatic events followed with trauma-response symptoms and depressive symptomatology remained significant even after controlling for social support. Therefore, it is possible that participants who experienced a traumatic event without further symptoms were able to benefit from other psychological resources (e.g., the ability to develop adoptive coping mechanisms and/or attribute positive meaning to the traumatic event) that helped them recover from the traumatic event faster, compared to those who experienced further symptoms (Bonanno 2005; Eagle and Kaminer 2015; Helitzer et al. 2014). High levels of resilience were shown to be associated with better mental health among older adults from diverse populations, including American Indians (Schure et al. 2013). More research focusing on the relationship between coping mechanisms and resilience in older American Indians who have experienced traumatic events is needed to better understand the trauma response among this population.

Several other tribal-level studies documented the negative mental health consequences of traumatic and/or stressful life events among older American Indians (Bassett et al. 2014; Beals et al. 2013; Roh et al. 2014). For example, among a sample of older American Indians from the Great Lakes region, stressful life events (some of which were potentially traumatic) were shown to be significant predictors for depression (Chapleski et al. 2004). Similarly, Roh et al. (2014) found a positive association between adverse childhood experiences and depressive symptoms among a sample of older American Indians residing in South Dakota and Minnesota. Our study contributes to this literature further, by including different levels of trauma response in the analysis, rather than simply assessing for the presence of a lifetime traumatic event. Using the same measure we used in this study, Marshall and colleagues found significant increases in psychological impairment, suicidal ideation, and major depressive disorder with each incremental increase in the number of trauma-response symptoms
(Marshall et al. 2001). Other studies have found positive associations between subthreshold PTSD and increases in healthcare utilization, work-loss days, and interpersonal withdrawal (Breslau et al. 2004). In our study, we were careful not to classify those who experienced a traumatic event followed with trauma-response symptoms as having subthreshold PTSD. Definitions of subthreshold PTSD vary, and it is not clear when those who experience a traumatic event with partial response symptoms are at greater risk for negative mental health outcomes. Because of samplesize limits, our categorization of experiences of trauma and the resulting symptoms was more liberal. That is, only one symptom item needed to be confirmed for a categorization of trauma-response symptoms, yet we still found a significant association with clinically relevant depressive symptomatology. There is a need for research that will further examine the level of impairment associated with different levels of trauma-response symptoms (Breslau et al. 2004; Cukor et al. 2010). This is especially true for the members of American Indian tribes, because diagnostic categories such as PTSD have limitations in identifying the impact of cumulative and community level traumatic events that American Indians have been experiencing over generations (Evans-Campbell 2008). Additionally, American Indian populations define and experience the concept of mental wellness in substantially different ways compared to other populations in the US (Evans-Campbell 2008; Moorehead et al. 2015). Acknowledging partial trauma responses among older American Indian adults might help reduce the unmet need for mental health services in this population, as it will increase access to treatment among those who fall short of the criteria for full PTSD but still experience psychological impairment (Cukor et al. 2010).

Trauma response is shaped by social- and cultural-level factors, which might play an important role in American Indian populations (Evans-Campbell 2008). For example, the historical context of the traumatic event, and culturally specific meanings associated with the traumatic event might affect the trauma response (Evans-Campbell 2008; Hartmann and Gone 2014). Traumatic events that are associated with collective identity, such as those due to systematic assimilation policies, can have prolonged effects on victims and their children (Brave Heart et al. 2012; Evans-Campbell 2008; Hartmann and Gone 2014; Whitbeck et al. 2004). Characteristics of the traumatic event, such as type, intensity, occurrence time, duration and frequency, also play an important role in shaping individual responses (Beals et al. 2013; Evans-Campbell 2008; Frans et al. 2005). Beals and colleagues examined the conditional risk of PTSD in two American Indian reservations and found higher rates of PTSD among participants who reported interpersonal events as their worst trauma, whereas participants who reported non-interpersonal trauma, were a witness to trauma or had a 
family member or friend experience trauma had lower rates of PTSD. Frans and Fredrikson (2005) found the highest risk of PTSD among those with exposure to multiple traumatic events (Frans et al. 2005). A better understanding of these factors in the context of older American Indians residing on tribal lands would help researchers identify the mechanisms through which traumatic events lead to diverse mental health outcomes.

Our study has several limitations. We are not able to conclude that experiences of trauma over the life course cause depressive symptomatology for several reasons. First, we do not know whether the traumatic event happened before, during or after the report of depressive symptomatology. Although depressive symptomatology is a common result of experiencing a traumatic event, future work should consider the timing of the traumatic event in relation to the depressive symptomatology to better understand causal mechanisms. Our data do not include the specific nature or number of traumatic events that the participants experienced. This additional information would be relevant to collect in future research, as a person's response to trauma is often shaped by the type, intensity, and frequency of the traumatic event experienced (Beals et al. 2013; Frans et al. 2005). Lastly, our trauma measure was self-reported and had a long recall period, which could increase recall bias.

Despite its limitations, our study adds to the literature in unique ways. It is among the few to examine the association between trauma with further symptoms and depressive symptomatology in a representative sample of older American Indians belonging to one tribe. This particular method is important because it takes into consideration the heterogeneity among tribes, which is shaped by different lifestyles, cultural traditions, healthcare and socioeconomic resources (Gone and Trimble 2012a; Hartmann and Gone 2014), while providing estimates that are generalizable to one tribal community. In addition, we adjusted for variables that could confound the association between experiences of trauma and depressive symptomatology, which should result in less biased results. Finally, our study used a rigorous data collection procedure with previously tested scales and items.

Our findings have implications for informing mental health providers, and public health professionals who serve older American Indians (Brave Heart et al. 2012; Cribbs et al. 2009). A "trauma-informed approach," which is suggested for developing effective community-based interventions dealing with trauma, posits that effective recovery from trauma is possible through three pathways: (1) acknowledging trauma and its impact on mental health; (2) recognizing the symptoms of trauma; and (3) responding to trauma on multiple levels (e.g., individual, community and policy) (Evans-Campbell 2008; Gone and Trimble 2012b). This approach is in line with recent work that the Substance Abuse and Mental Health Services Administration
(SAMSHA) has done in collaboration with the Indian Health Services and the National Indian Health Board that seeks to create support systems, increase community connectedness, and break the cycle of historical and intergenerational trauma in American Indian populations (Substance Abuse and Mental Health Services Administration, Indian Health Services and The National Indian Health Board 2016). Native scholars offered an extension to this definition by incorporating cultural context of each American Indian tribe into the diagnosis and treatment of trauma-related disorders among tribal members (Moghaddam et al. 2015; Roh et al. 2015). Informed by this theoretical background, our results suggest that mental health providers who serve older American Indians should consider the experience of traumatic events followed with response symptoms as a potential risk factor for further disruptions in psychological functioning. Incorporating this knowledge into the clinical screening processes applied in older American Indians would be useful, as they might benefit from culturally-tailored and traumainformed treatment options even if they do not confirm full or subthreshold PTSD criteria.

Acknowledgements This research was sponsored by the National Institute on Aging (AG022336). The views expressed in this article are those of the authors and do not represent the U.S. Department of Agriculture, Food and Nutrition Service.

\section{Compliance with Ethical Standards}

Conflict of interest The authors do not have any conflicts that would influence the findings.

\section{References}

Bassett, D., Buchwald, D., \& Manson, S. (2014). Posttraumatic stress disorder and symptoms among American Indians and Alaska natives: A review of the literature. Social Psychiatry and Psychiatric Epidemiology, 49(3), 417-433. https://doi.org/10.1007/ s00127-013-0759-y.

Beals, J., Belcourt-Dittloff, A., Garroutte, E. M., Croy, C., Jervis, L. L., Whitesell, N. R., ... Manson, S. M. (2013). Trauma and conditional risk of posttraumatic stress disorder in two American Indian reservation communities. Social Psychiatry and Psychiatric Epidemiology, 48(6), 895-905. https://doi.org/10.1007/ s00127-012-0615-5.

Beals, J., Novins, D. K., Whitesell, N. R., Spicer, P., Mitchell, C. M., \& Manson, S. M. (2005). Prevalence of mental disorders and utilization of mental health services in two American Indian reservation populations: Mental health disparities in a national context. The American Journal of Psychiatry, 162(9), 1723-1732. https://doi. org/10.1176/appi.ajp.162.9.1723.

Bonanno, G. A. (2005). Resilience in the face of loss and potential trauma. Current Directions in Psychological Science, 14(3), 135138. https://doi.org/10.1111/j.0963-7214.2005.00347.x.

Brave Heart, M. Y. H., Elkins, J., Tafoya, G., Bird, D., \& Salvador, M. (2012). Wicasa Was'aka: Restoring the traditional strength of American Indian boys and men. American Journal of Public Health. https://doi.org/10.2105/AJPH.2011.300511. 
Breslau, N., Lucia, V. C., \& Davis, G. C. (2004). Partial PTSD versus full PTSD: An empirical examination of associated impairment. Psychological Medicine, 34(7), 1205-1214. https://doi. org/10.1017/S0033291704002594.

Chapleski, E. E., Kaczynski, R., Gerbi, S. A., \& Lichtenberg, P. A. (2004). American Indian elders and depression: Short-and longterm effects of life events. Journal of Applied Gerontology, 23(1), 40-57. https://doi.org/10.1177/0733464804263087.

Chapleski, E. E., Lamphere, J. K., Kaczynski, R., Lichtenberg, P. A., \& Dwyer, J. W. (1997). Structure of a depression measure among American Indian elders: Confirmatory factor analysis of the CES-D Scale. Research on Aging. https://doi. org/10.1177/0164027597194004.

Cribbs, J. M., Byers, L., \& Moxley, D. (2009). Serving older native Americans: Challenges facing gerontological social work in Indian Country. Journal of Ethnic And Cultural Diversity in Social Work. https://doi.org/10.1080/15313200903310734.

Cukor, J., Wyka, K., Jayasinghe, N., \& Difede, J. (2010). The nature and course of subthreshold PTSD. Journal of Anxiety Disorders, 24(8), 918-923. https://doi.org/10.1016/j.janxdis.2010.06.017.

Eagle, G. T., \& Kaminer, D. (2015). Traumatic stress: established knowledge, current debates and new horizons. South African Journal of Psychology, 45(1), 22-36. https://doi. org/10.1177/0081246314547124.

Ehlers, C. L., Gizer, I. R., Gilder, D. A., \& Yehuda, R. (2013). Lifetime history of traumatic events in an American Indian community sample: Heritability and relation to substance dependence, affective disorder, conduct disorder and PTSD. Journal of Psychiatric Research, 47(2), 155-161. https://doi.org/10.1016/j. jpsychires.2012.10.002.

Evans-Campbell, T. (2008). Historical trauma in American Indian/ Native Alaska communities: A multilevel framework for exploring impacts on individuals, families, and communities. Journal of Interpersonal Violence, 23(3), 316-338. https://doi. org/10.1177/0886260507312290.

Frans, O., Rimmö, P., Aberg, L., \& Fredrikson, M. (2005). Trauma exposure and post-traumatic stress disorder in the general population. Acta Psychiatrica Scandinavica, 111(4), 291-299. https:// doi.org/10.1111/j.1600-0447.2004.00463.x.

Gameiro, G. R., Minguini, I. P., Correa, T., \& Ferraz, D. T. (2014). The role of stress and life events in the onset of depression in the elderly O papel do estresse e de acontecimentos cotidianos no desenvolvimento da depressão na terceira idade. Rev Med (São Paulo), 93(1), 31-40.

Goins, R. T., Garroutte, E. M., Fox, S. L., Dee Geiger, S., \& Manson, S. M. (2011). Theory and practice in participatory research: Lessons from the native elder care study. The Gerontologist, 51(3), 285-294. https://doi.org/10.1093/geront/gnq130.

Gone, J. P., \& Trimble, J. E. (2012a). American Indian and Alaska native mental health: Diverse perspectives on enduring disparities. Annual Review of Clinical Psychology. https://doi.org/10.1146/ annurev-clinpsy-032511-143127.

Gone, J. P., \& Trimble, J. E. (2012b). American Indian and Alaska Native mental health: diverse perspectives on enduring disparities. Annual Review of Clinical Psychology, 8, 131-160. https:// doi.org/10.1146/annurev-clinpsy-032511-143127.

Goodkind, J. R., Ross-Toledo, K., John, S., Hall, J. L., Ross, L., Freeland, L., ... Lee, C. (2010). Promoting healing and restoring trust: Policy recommendations for improving behavioral health care for American Indian/Alaska Native Adolescents. American Journal of Community Psychology, 46(3), 386-394. https://doi.org/10.1007/ s10464-010-9347-4.

Hartmann, W. E., \& Gone, J. P. (2014). American Indian historical trauma: Community perspectives from two great plains medicine men. American Journal of Community Psychology, 54(3-4), 274-288. https://doi.org/10.1007/s10464-014-9671-1.
Helitzer, D., Graeber, D., LaNoue, M., \& Newbill, S. (2014). Don't step on the tiger's tail: A mixed methods study of the relationship between adult impact of childhood adversity and use of coping strategies. Community Mental Health Journal, 51(7), 768-774. https://doi.org/10.1007/s10597-014-9815-7.

Inouye, S. K., Robison, J. T., Froehlich, T. E., \& Richardson, E. D. (1998). The time and change test: A simple screening test for dementia. The Journals of Gerontology Series A: Biological Sciences and Medical Sciences, 53A(4), M281-M286. https:// doi.org/10.1093/gerona/53A.4.M281.

Lillie-Blanton, M., \& Roubideaux, Y. (2005). Understanding and addressing the health care needs of American Indians and Alaska Natives. American Journal of Public Health, 95(5), 759-761. https://doi.org/10.2105/AJPH.2005.063230.

Manson, S. M., Beals, J., Klein, S. A., \& Croy, C. D. (2005). Social epidemiology of trauma among 2 American Indian reservation populations. American Journal of Public Health, 95(5), 851859. https://doi.org/10.2105/AJPH.2004.054171.

Marshall, R. D., Olfson, M., Hellman, F., Blanco, C., Guardino, M., \& Struening, E. L. (2001). Comorbidity, impairment, and suicidality in subthreshold PTSD. American Journal of Psychiatry, 158(9), 1467-1473. https://doi.org/10.1176/appi. ajp.158.9.1467.

Moghaddam, J. F., Momper, S. L., \& Fong, W., T (2015). Crystalizing the role of traditional healing in an urban Native American Health Center. Community Mental Health Journal, 51(3), 305-314. https://doi.org/10.1007/s10597-014-9813-9.

Moorehead, V. D., Gone, J. P., \& December, D. (2015). A gathering of native American healers: Exploring the interface of indigenous tradition and professional practice. American Journal of Community Psychology, 56(3-4), 383-394. https://doi.org/10.1007/ s10464-015-9747-6.

Radloff, L. S. (1977). The CES-D Scale: A Self-Report Depression Scale for Research in the General Population. Applied Psychological Measurement. https://doi.org/10.1177/014662167700100306.

Ritsher, J. B., Struening, E. L., Hellman, F., \& Guardino, M. (2002). Internal validity of an anxiety disorder screening instrument across five ethnic groups. Psychiatry Research, 111(2-3), 199213. https://doi.org/10.1016/S0165-1781(02)00135-X.

Robin, R. W., Chester, B., Rasmussen, J. K., Jaranson, J. M., \& Goldman, D. (1997). Prevalence and characteristics of trauma and posttraumatic stress disorder in a southwestern American Indian community. The American Journal of Psychiatry, 154(11), 1582-1588. Retrieved from http://www.ncbi.nlm.nih.gov/ pubmed/9356568.

Roh, S., Brown-Rice, K. A., Lee, K. H., Lee, Y.-S., Lawler, M. J., \& Martin, J. I. (2015a). Stressors, coping resources, and depressive symptoms among rural American Indian older adults. Social Work in Public Health, 30(4), 345-359. https://doi.org/10.1080/19371 918.2015.1019174.

Roh, S., Brown-Rice, K. A., Lee, K. H., Lee, Y.-S., Yee-Melichar, D., \& Talbot, E. P. (2015b). Attitudes toward mental health services among American Indians by two age groups. Community Mental Health Journal. https://doi.org/10.1007/s10597-015-9859-3.

Roh, S., Burnette, C. E., Lee, K. H., Lee, Y.-S., Easton, S. D., \& Lawler, M. J. (2014). Risk and protective factors for depressive symptoms among American Indian older adults: Adverse childhood experiences and social support. Aging \& Mental Health, 19(4), 371-380. https://doi.org/10.1080/13607863.2014.938603.

Roth, R. S., Geisser, M. E., \& Bates, R. (2008). The relation of posttraumatic stress symptoms to depression and pain in patients with accident-related chronic pain. The Journal of Pain: Official Journal of the American Pain Society, 9(7), 588-596. https://doi. org/10.1016/j.jpain.2008.01.333.

Schure, M., \& Goins, R. T. Psychometric examination of the Center for Epidemiologic Studies Depression scale with older American 
Indians: The Native Elder Care Study. American Indian and Alaska Native Mental Health Research. (in press)

Schure, M. B., Odden, M., \& Goins, R. T. (2013). The association of resilience with mental and physical health among older American Indians: The native elder care study. American Indian and Alaska Native Mental Health Research, 20(2), 27-41. Retrieved from http://www.ncbi.nlm.nih.gov/pubmed/3805026.

Sherbourne, C. D., \& Stewart, A. L. (1991). The MOS social support survey. Social Science \& Medicine, 32(6), 705-714. https://doi. org/10.1016/0277-9536(91)90150-B.

Stein, M. B., Walker, J. R., Hazen, A. L., \& Forde, D. R. (1997). Full and partial posttraumatic stress disorder: Findings from a community survey. American Journal of Psychiatry, 154(8), 1114-1119.

Substance Abuse and Mental Health Services Administration, Indian Health Services, \& The National Indian Health Board. (2016). The
National Tribal Behavioral Health Agenda. Retrieved from https:// store.samhsa.gov/product/PEP16-NTBH-AGENDA.

Von Hippel, P. T. (2007). Regression with missing Ys: An improved strategy for analyzing multiply imputed data. Sociological Methodology, 37(1), 83-117. https://doi. org/10.1111/j.1467-9531.2007.00180.x.

Whitbeck, L. B., Adams, G. W., Hoyt, D. R., \& Chen, X. (2004). Conceptualizing and measuring historical trauma among American Indian people. American Journal of Community Psychology, 33(3-4), 119-130. https://doi.org/10.1023/B:A JCP.0000027000.77357.31.

White, I. R., Royston, P., \& Wood, A. M. (2011). Multiple imputation using chained equations: Issues and guidance for practice. Statistics in Medicine, 30(4), 377-399. https://doi.org/10.1002/ sim.4067. 